\title{
Stable Zinc Anodes Enabled by Zincophilic Cu Nanowire Networks
}

Cite as

Nano-Micro Lett.

(2022) $14: 39$

Received: 2 October 2021

Shiyin Xie ${ }^{1}$, Yang $\mathrm{Li}^{1}, \mathrm{Xu} \mathrm{Li}^{1}$, Yujun Zhou ${ }^{1}$, Ziqi Dang ${ }^{1}$, Jianhua Rong ${ }^{1}$,

Accepted: 1 December 2021

Published online: 23 December 2021

(C) The Author(s) 2021

\section{HIGHLIGHTS}

- A novel strategy of employing $\mathrm{Cu}$ nanowire networks to realize superior long-life and high-rate zinc anodes was proposed.

- $\mathrm{Cu}$ nanowire networks could stabilize zinc anodes from multiple aspects, including homogenizing surface electric field and $\mathrm{Zn}^{2+}$ concentration field, inhibiting side reactions and inducing uniform zinc nucleation/deposition.

- Facets and edge sites of $\mathrm{Cu}$ nanowires, especially the latter ones, were revealed to be highly zincophilic.

\begin{abstract}
Zn-based electrochemical energy storage (EES) systems have received tremendous attention in recent years, but their zinc anodes are seriously plagued by the issues of zinc dendrite and side reactions (e.g., corrosion and hydrogen evolution). Herein, we report a novel strategy of employing zincophilic $\mathrm{Cu}$ nanowire networks to stabilize zinc anodes from multiple aspects. According to experimental results, COMSOL simulation and density functional theory calculations, the $\mathrm{Cu}$ nanowire networks covering on zinc anode surface not only homogenize the surface electric field and $\mathrm{Zn}^{2+}$ concentration field, but also inhibit side reactions through their hydrophobic feature. Meanwhile, facets and edge sites of the $\mathrm{Cu}$ nanowires, especially the latter ones, are revealed to be highly zincophilic to induce uniform zinc nucleation/deposition. Consequently, the $\mathrm{Cu}$ nanowire networks-
\end{abstract}

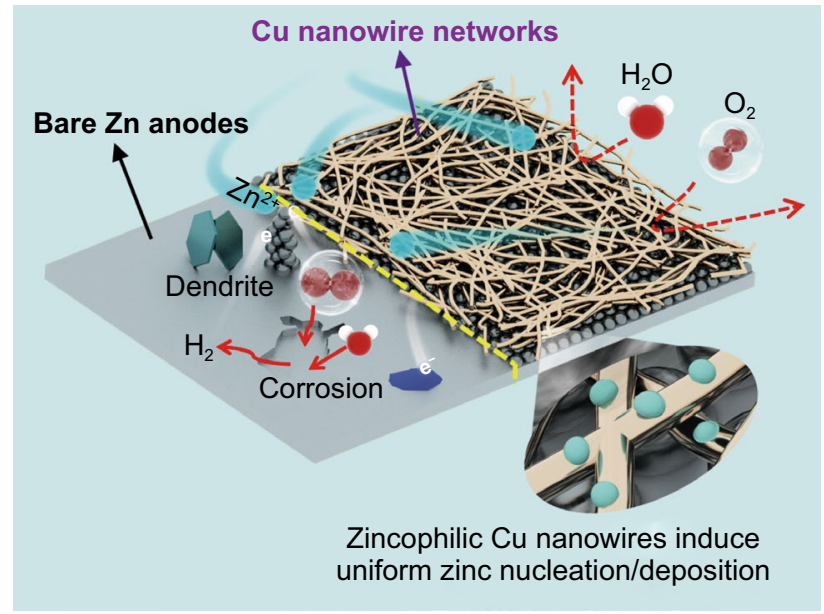
protected zinc anodes exhibit an ultralong cycle life of over $2800 \mathrm{~h}$ and also can continuously operate for hundreds of hours even at very large charge/discharge currents and areal capacities (e.g., $10 \mathrm{~mA} \mathrm{~cm}{ }^{-2}$ and $5 \mathrm{mAh} \mathrm{cm}^{-2}$ ), remarkably superior to bare zinc anodes and most of currently reported zinc anodes, thereby enabling Zn-based EES devices to possess high capacity, 16,000-cycle lifespan and rapid charge/discharge ability. This work provides new thoughts to realize long-life and high-rate zinc anodes.

KEYWORDS Zn-based energy storage; Zinc anodes; Zinc dendrite; Zincophilic materials; Cu nanowire networks

Shiyin Xie and Yang Li have contributed equally to this work.

$\triangle$ Liubing Dong, donglb@jnu.edu.cn

1 College of Chemistry and Materials Science, Jinan University, Guangzhou 511443, People's Republic of China 


\section{Introduction}

The rapid development of electric vehicles, wearable electronic products and smart grids has aroused enthusiasm for seeking safe, high-energy, low-cost and environmentally friendly electrochemical energy storage (EES) systems. Among various EES systems, aqueous Zn-based EES systems using neutral or mildly acidic electrolytes, typified by zinc-ion batteries and zinc-ion hybrid supercapacitors, have received tremendous attention in recent years [1-5]. Application of aqueous electrolytes with high ionic conductivity endows the Zn-based EES systems with high safety, low toxicity and the potential for realizing fast charge/discharge. Meanwhile, metallic zinc anodes in the Zn-based EES systems are characterized by high theoretical capacity $\left(820 \mathrm{mAh} \mathrm{g}^{-1}\right.$ and 5,845 $\left.\mathrm{Ah} \mathrm{L}^{-1}\right)$ and relatively low electrochemical potential $(-0.762 \mathrm{~V}$ vs. standard hydrogen potential), being beneficial for the $\mathrm{Zn}$ based EES systems to achieve high energy density [6-8]. Moreover, diverse cathode materials such as manganese oxides, vanadium oxides, carbon materials, Prussian blue analogs and organic materials have been explored for aqueous Zn-based EES [9-17], and encouragingly, electrochemical performance of cathode materials for aqueous Zn-based EES devices is continuously improved.

Despite the above merits of aqueous Zn-based EES systems, their practical application is hampered by the electrochemical and thermodynamic instability of metallic zinc anodes [18-20]. To be specific, during zinc plating/stripping processes, the microscopic surface of zinc anodes changes continuously, thereby causing an inhomogeneous electric field (e.g., protuberance sites generally create a strong localized electrical field due to the "tip effect") [21]. The inhomogeneous surface electric field, coupling with rampant 2D diffusion of $\mathrm{Zn}^{2+}$ on the zinc anode surface, raises the issues of zinc dendrites and short circuits [21,22]. At large charge/ discharge currents, these issues become more serious [23]. Meanwhile, the mildly acidic feature and dissolved oxygen of aqueous zinc salt electrolytes such as $\mathrm{ZnSO}_{4}$ solutions, as well as relatively high hydrogen evolution potential (vs. $\mathrm{Zn}^{2+} / \mathrm{Zn}$ redox potential), often result in corrosion of metallic zinc and hydrogen evolution reactions, accelerating the failure of zinc anodes [24, 25]. Therefore, aqueous Zn-based EES devices suffer from low coulombic efficiency, unsatisfactory rate performance and inferior cycle lifetime.
To stabilize zinc anodes, several strategies have been proposed such as anode structure design and anode surface modification. For instance, employing a 3D conductive skeleton as the current collector for zinc anodes is beneficial for reducing local current density and thus alleviating the serious aggregation of electrons and $\mathrm{Zn}^{2+}$ in particular positions [26, 27]. Artificial interface layers (e.g., porous inorganic particle coating, metal-organic framework layer or polymer coating) introduced on zinc anode surface are capable of guiding $\mathrm{Zn}^{2+}$ flux to guarantee uniform deposition of zinc, as well as blocking free water with dissolved oxygen to avoid corrosion of zinc anodes [22, 28-32], but the huge impedance of interfaces between these nonconductive artificial layers and zinc anodes is against rate capability of Zn-based EES devices [33]. Furthermore, when conductive and porous carbon networks are utilized as the artificial interface layers, they may synchronously regulate $\mathrm{Zn}^{2+}$ flux and maintain a stable electric field on zinc anode surface to optimize zinc plating/stripping behaviors [34, 35]. Besides the above strategies, very recent research studies found that zinc plating on specific substrates shows low nucleation barriers and fast kinetics [36-40]. In such cases, $\mathrm{Zn}^{2+}$ ions are easy to be captured by zincophilic sites on these substrates and react with electrons to form zinc atoms/clusters, and in return, the number of electrons and $\mathrm{Zn}^{2+}$ ions accumulated at anode/electrolyte interfaces is reduced, thereby effectively mitigating zinc dendrite growth. This provides a new approach for stabilizing zinc anodes, but only very limited types of zincophilic materials have been investigated, and meanwhile, some zincophilic materials such as $\mathrm{N}$-doped carbon may catalyze water decomposition which is undesired [41]. In addition, the zinc plating process is composed of several successive steps such as electrostatic adsorption of $\mathrm{Zn}^{2+}$ and zinc nucleation. However, many of the current strategies mainly focus on modulating one of the above steps to stabilize zinc anodes. For instance, porous inorganic coating on zinc anodes can guide $\mathrm{Zn}^{2+}$ flux [32], but hardly induces zinc nucleation; using $\mathrm{Cu}$ plates/foams as current collectors can reduce nucleation barriers of zinc deposition [26], but cannot effectively reduce the local current density due to their limited specific surface area [42, 43]. In theory, a comprehensive strategy that can positively affect both $\mathrm{Zn}^{2+} /$ electron concentration distribution and zinc nucleation is expected to endow zinc anodes with better electrochemical performance. 
Herein, we report a strategy of employing zincophilic $\mathrm{Cu}$ nanowire networks to stabilize zinc anodes from multiple aspects. The $\mathrm{Cu}$ nanowire networks-protected zinc anodes exhibit stable plating/stripping behaviors at various charge/ discharge currents and areal capacities, significantly superior to bare zinc anodes and most of currently reported zinc anodes. According to experimental results and COMSOL simulation, for the $\mathrm{Cu}$ nanowire networks covering on zinc anode surface, their porous nature favors uniform $\mathrm{Zn}^{2+}$ flux, and meanwhile, nanoscale size endows the $\mathrm{Cu}$ nanowires with large specific surface area, which helps to reduce local current density and homogenize $\mathrm{Zn}^{2+}$ concentration field, thereby restraining zinc dendrite growth. Besides, by virtue of hydrophobic feature, the $\mathrm{Cu}$ nanowire networks suppress the direct contact between free water (with dissolved oxygen) and zinc anodes, which inhibits side reactions such as anode corrosion and hydrogen evolution. Density functional theory (DFT) calculations reveal that the facets and edge sites of the $\mathrm{Cu}$ nanowires, especially the latter ones, are highly zincophilic to induce uniform nucleation/deposition of zinc. Moreover, electrochemical stability of the $\mathrm{Cu}$ nanowire networks-protected zinc anodes is further examined in $\mathrm{Zn}$-based EES devices.

\section{Experimental Section}

\subsection{Material Preparation}

\subsubsection{Preparation of Cu Nanowire Networks}

$0.17 \mathrm{~g} \mathrm{CuCl}_{2} \cdot 2 \mathrm{H}_{2} \mathrm{O}$ and $0.308 \mathrm{~g}$ glucose were dissolved in $50 \mathrm{~mL}$ deionized water, and then $1.44 \mathrm{~g}$ hexadecylamine was slowly added to the above solution to get a homogeneous light blue emulsion after continuous magnetic stirring for $12 \mathrm{~h}$. The emulsion was transferred to a $100-\mathrm{mL}$ Teflon-lined autoclave and heated at $120^{\circ} \mathrm{C}$ for $6 \mathrm{~h}$. After cooling down to room temperature naturally, precipitation products were washed with hexane/ethanol mixture solution (2: 1 in volume) five times through the centrifugation method, followed by washing with deionized water and freeze-drying to obtain $\mathrm{Cu}$ nanowire powder. $5 \mathrm{mg}$ of $\mathrm{Cu}$ nanowire powder was dispersed in $5 \mathrm{~mL}$ of anhydrous ethanol by the ultrasonic dispersion method, and $50 \mathrm{~mL}$ of deionized water was added to get a uniform suspension. The suspension was vacuumfiltered through a hydrophilic filter membrane with a pore size of $0.8 \mu \mathrm{m}$ to fabricate $\mathrm{Cu}$ nanowires-coated membranes (denoted as "CuNW/membranes"), in which $\mathrm{Cu}$ nanowires $\left(0.4 \mathrm{mg} \mathrm{cm}^{-2}\right.$ ) randomly dispersed on the membrane to form $\mathrm{Cu}$ nanowire networks. The $\mathrm{CuNW} /$ membranes were cut into disks with a diameter of $10 \mathrm{~mm}$.

\subsubsection{Synthesis of Hydrous Ruthenium Oxide/Graphene (GR) Nanocomposite Cathode Material}

$5.24 \mathrm{~mL}$ of graphene oxide aqueous gel (1.24 wt\%, produced by Aladdin Reagent Co. Ltd., China) and $286.6 \mathrm{mg}$ $\mathrm{RuCl}_{3} \cdot 3 \mathrm{H}_{2} \mathrm{O}$ were mixed with $20 \mathrm{~mL}$ of deionized water under magnetic stirring and transferred to a 50-mL Teflonlined autoclave, followed by heating at $180{ }^{\circ} \mathrm{C}$ for $6 \mathrm{~h}$ and naturally cooling down to room temperature. GR nanocomposite was obtained by washing the precipitation products with deionized water and drying overnight at $80^{\circ} \mathrm{C}$.

\subsection{Material Characterizations}

X-ray diffraction (XRD) tests were performed on a diffraction analyzer (model: Rigaku MiniFlex 600) with $\mathrm{Cu} \mathrm{K \alpha}$ $(\lambda=0.15418 \mathrm{~nm})$ radiation. Micro-morphologies of samples were observed using field emission scanning electron microscopy (SEM) (model: Zeiss Sigma 300) and transmission electron microscopy (TEM) (model: FEI Tecnai G2 F20). Thermogravimetric (TG) measurement was carried out on a simultaneous thermal analyzer (model: TGA2) from 30 to $700{ }^{\circ} \mathrm{C}$ with a heating rate of $5{ }^{\circ} \mathrm{C} \mathrm{min}{ }^{-1}$ under air atmosphere. Water contact angle tests were performed on a contact angle tester (model: DSA-100).

\subsection{Electrochemical Measurements}

Electrochemical behaviors of zinc anodes with and without the protection of $\mathrm{Cu}$ nanowire networks were studied by assembling symmetric CR2032 coin cells, in which non-woven cloth separator and $2 \mathrm{M} \mathrm{ZnSO}_{4}$ aqueous electrolyte were utilized. For unprotected zinc anodes (i.e., bare $\mathrm{Zn}$ anodes), zinc foil disks were directly used as the electrodes of symmetric cells, while in symmetric cells with $\mathrm{Cu}$ nanowire networks-protected zinc anodes, a piece of $\mathrm{CuNW} / \mathrm{membrane}$ was placed between each zinc foil disk electrode and the separator, and $\mathrm{Cu}$ nanowirescoated side of the $\mathrm{CuNW} / \mathrm{membranes}$ contacted with zinc 
foils (i.e., the filter membrane substrate of the CuNW/ membranes directly contacted with the separator). The zinc foils with a thickness of $70 \mu \mathrm{m}$ were purchased from Qinghe Haoxuan Metal Materials Co. Ltd., China. The $\mathrm{Cu}$ nanowire networks-protected zinc anodes are briefly denoted as "Zn@CuNW" to differentiate them from bare $\mathrm{Zn}$ anodes in the following. Rate performance and cycling stability of the symmetric cells were evaluated using galvanostatic charge-discharge (GCD) technique at different current densities $\left(0.1-10 \mathrm{~mA} \mathrm{~cm}^{-2}\right)$ and areal capacities (0.1-10 $\mathrm{mAh} \mathrm{cm}^{-2}$ ) on a LAND CT2001A battery testing instrument. Linear polarization and chronoamperometry tests of the bare $\mathrm{Zn}$ anodes and $\mathrm{Zn@CuNW}$ anodes were performed on an electrochemical workstation (model: CHI760E).

Zinc-ion hybrid supercapacitors were assembled with zinc anodes, GR nanocomposite cathodes and $2 \mathrm{M} \mathrm{ZnSO}_{4}$ aqueous electrolyte. To prepare the GR composite cathodes, the GR nanocomposite active material, acetylene black and polyvinylidene fluoride, in a mass ratio of $7: 2: 1$, were mixed with $\mathrm{N}$-methylpyrrolidone and then coated on stainless steel foil, followed by complete drying. Cyclic voltammetry (CV) curves and electrochemical impedance spectroscopy (EIS) spectra (with a frequency range of $0.01 \mathrm{~Hz}$ to $100 \mathrm{kHz}$ and an amplitude of $5 \mathrm{mV}$ at open-circuit voltage state) of the zinc-ion hybrid supercapacitors were recorded on the CHI760E electrochemical workstation. GCD tests at current densities of 0.1-1 A $\mathrm{g}^{-1}$ were performed on a LAND CT2001A battery testing instrument, while those at $2-20 \mathrm{~A} \mathrm{~g}^{-1}$ were carried out on the CHI760E electrochemical workstation (with higher precision to guarantee the accuracy of testing data).

\subsection{Theoretical Calculation and Simulation}

COMSOL Multiphysics finite element method was used to theoretically simulate electric field and $\mathrm{Zn}^{2+}$ concentration distribution on zinc anode surface during zinc plating processes. Spin-polarized DFT calculations were employed to investigate the adsorption energy of single zinc atom on various substrates, e.g., different facets and edge sites of $\mathrm{Cu}$ nanowires. Detailed procedures of the COMSOL simulation and DFT calculations are provided in Supporting Information.

\section{Results and Discussion}

\subsection{Material Characterizations}

Physicochemical characteristics of the synthesized $\mathrm{Cu}$ nanowire sample are shown in Fig. 1. In the XRD pattern (Fig. 1a), the three diffraction peaks at $2 \theta=43.3^{\circ}, 50.4^{\circ}$ and $74.2^{\circ}$ are indexed to the (111), (200) and (220) crystal planes of face-centered cubic metallic Cu (JCPDS \#04-0836). TEM observation shows that most $\mathrm{Cu}$ nanowires have a diameter of 40-60 nm and a length of several micrometers (Fig. 1b). Selected area electron diffraction reveals single-crystalline nature of the $\mathrm{Cu}$ nanowires (Fig. S1). High-resolution TEM image in Fig. 1c displays interplanar spacing of $0.21 \mathrm{~nm}$ that is identified as the (111) crystal plane of the $\mathrm{Cu}$ nanowires. An aqueous suspension of the $\mathrm{Cu}$ nanowires was vacuumfiltered through a hydrophilic filter membrane, and then $\mathrm{Cu}$ nanowire-coated membranes (denoted as " $\mathrm{CuNW} / \mathrm{mem}$ brane") were obtained (Fig. 1d). For the CuNW/membranes, $\mathrm{Cu}$ nanowires disorderly disperse on the surface of the filter membrane to form networks whose thickness is $\sim 18 \mu \mathrm{m}$, as exhibited by SEM images in Figs. 1e and S2-S3. The Cu nanowire networks are highly hydrophobic, with a water contact angle of $146^{\circ}$ (Fig. 1f). By contrast, the water contact angle of bare $\mathrm{Zn}$ foils and the filter membrane is only $93^{\circ}$ and $34^{\circ}$, respectively (Figs. 1f and S4). This means that when the $\mathrm{Cu}$ nanowire networks are used to protect zinc anodes, they are expected to prevent free water and dissolved oxygen inherent in aqueous zinc salt electrolytes from contacting and corroding zinc anodes [22, 44], as illustrated in Fig. 1g.

\subsection{Electrochemical Performance}

To assess the stabilizing effects of the $\mathrm{Cu}$ nanowire networks on zinc anodes, symmetric cells with bare $\mathrm{Zn}$ foil electrodes (denoted as "bare $\mathrm{Zn}$ ") and the $\mathrm{Cu}$ nanowire networks-protected Zn foil electrodes (denoted as “Zn@ CuNW") were assembled (Fig. S5). We can see from Figs. 2a and S6 that Zn@CuNW electrode-based symmetric cells exhibit stable charge/discharge behaviors with small voltage hysteresis at various current densities of 0.1-5 $\mathrm{mA} \mathrm{cm}^{-2}$ and areal capacities of 0.1-5 $\mathrm{mAh}$ $\mathrm{cm}^{-2}$. By comparison, bare $\mathrm{Zn}$ electrode-based symmetric 

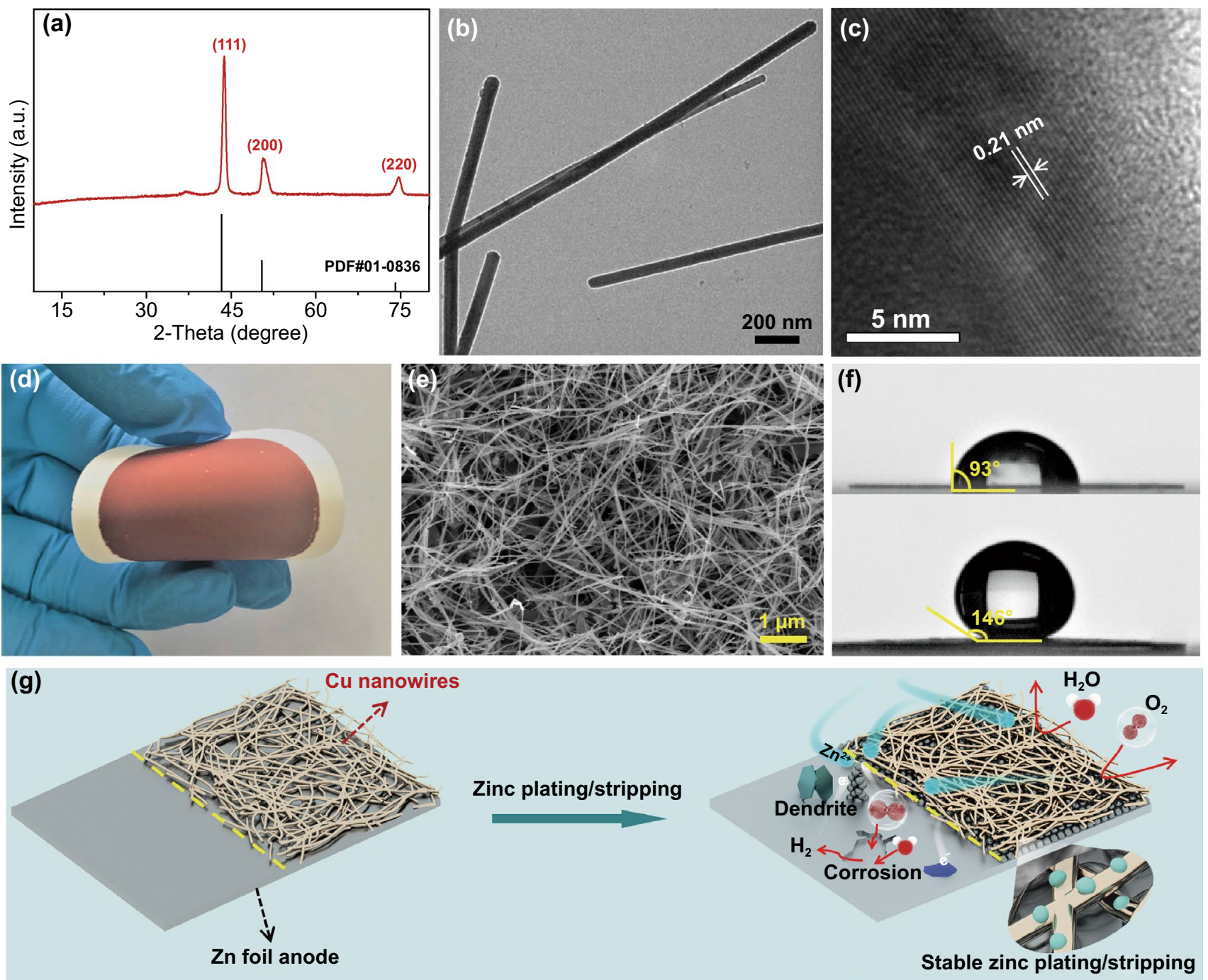

Fig. 1 a XRD pattern, $\mathbf{b}$ TEM and $\mathbf{c}$ high-resolution TEM images of the $\mathrm{Cu}$ nanowire sample. $\mathbf{d}$ Digital photograph and $\mathbf{e}$ surface SEM image of the $\mathrm{CuNW} / \mathrm{membrane.} \mathbf{f}$ Water contact angle of the CuNW/membrane (upper) and $\mathrm{Zn}$ foils (below). g Schematic illustration of protective effects of $\mathrm{Cu}$ nanowire networks on zinc anodes

cells show large voltage hysteresis, and abnormal charge/ discharge profiles at relatively large current densities and areal capacities. These prove that zinc plating/stripping on the Zn@CuNW electrodes possesses fast kinetics [32, 45], which is beneficial for realizing high-rate Zn-based EES. Figures $2 \mathrm{~b}$ and $\mathrm{S} 7$ show the long-term cycling stability of these symmetric cells at a current density of $0.2 \mathrm{~mA} \mathrm{~cm}^{-2}$ and an areal capacity of $0.2 \mathrm{mAh} \mathrm{cm}^{-2}$. Continuous operation time of Zn@CuNW electrode-based symmetric cells exceeds $2800 \mathrm{~h}$, much longer than that of symmetric cells with bare $\mathrm{Zn}$ electrodes (whose operation time is shorter than $100 \mathrm{~h}$ ), demonstrating that electrochemical stability of zinc anodes can be significantly improved under the protection of the $\mathrm{Cu}$ nanowire networks. Moreover, $\mathrm{Zn@}$ CuNW//Zn@CuNW symmetric cells display outstanding cycling performance at larger current densities and areal capacities (Figs. 2c-d and S8). Impressively, even at $10 \mathrm{~mA} \mathrm{~cm}{ }^{-2}$ and $5 \mathrm{mAh} \mathrm{cm}{ }^{-2}, \mathrm{Zn} @ \mathrm{CuNW} / / \mathrm{Zn} @ \mathrm{CuNW}$ symmetric cells are capable of operating over $130 \mathrm{~h}$, accompanying with stable charge/discharge behaviors and low voltage hysteresis of only $\sim 200 \mathrm{mV}$ (Fig. 2d). Such outstanding cycling stability and rate performance are not only notably superior to those of bare Zn electrode-based symmetric cells, but also have rarely been achieved in previous research studies (Table S1). Note that electrochemical properties of the $\mathrm{Cu}$ nanowire networks-protected zinc 

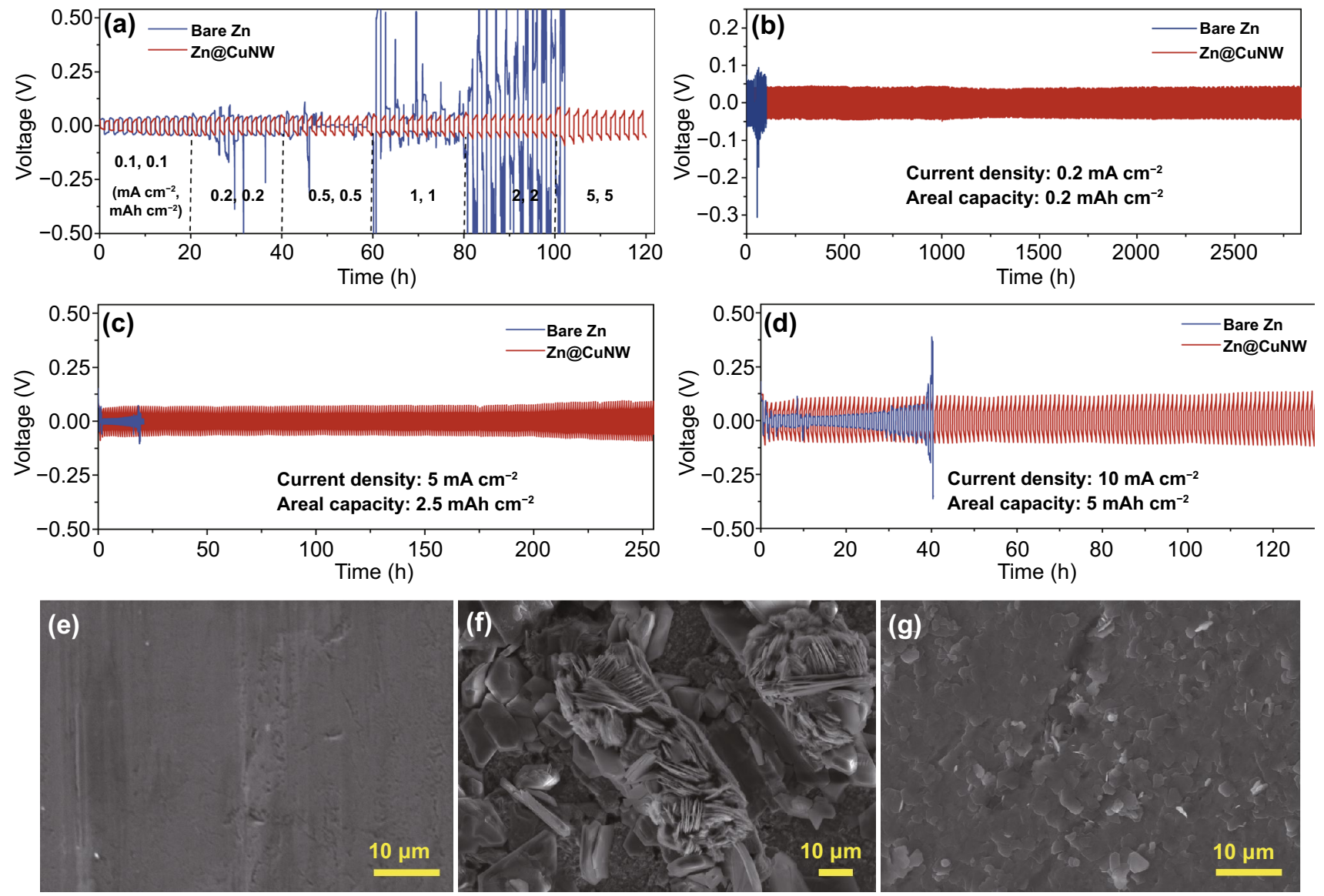

Fig. 2 a Rate performance of the symmetric cells with bare Zn foil electrodes and Zn@CuNW electrodes. Cycling stability of the symmetric

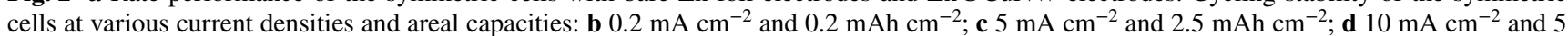
$\mathrm{mAh} \mathrm{cm}^{-2}$. SEM images of e pristine $\mathrm{Zn}$ foil, $\mathbf{f}$ bare $\mathrm{Zn}$ foil after cycling for $100 \mathrm{~h}$, and $\mathbf{g}$ protected $\mathrm{Zn}$ foil after cycling for $100 \mathrm{~h}$

anodes are affected by the mass loading of the $\mathrm{Cu}$ nanowires on the filter membranes (Fig. S9).

Furthermore, since failure of zinc anodes in aqueous zinc salt electrolytes is often caused by the issues of zinc dendrite, corrosion and hydrogen evolution (as illustrated in Fig. 1g) [7], we therefore observed the micro-morphologies of the bare $\mathrm{Zn}$ electrodes and the $\mathrm{Cu}$ nanowire networksprotected $\mathrm{Zn}$ electrodes after repeated zinc plating/stripping processes (i.e., these electrodes-based symmetric cells were charged/discharged for 50 and $100 \mathrm{~h}$ at a current density of $5 \mathrm{~mA} \mathrm{~cm}^{-2}$ and areal capacity of $2.5 \mathrm{mAh} \mathrm{cm}^{-2}$ ). As can be seen from Figs. 2e-g and S10, pristine $\mathrm{Zn}$ foils have a flat surface, but the bare $\mathrm{Zn}$ foil electrodes after cycling exhibit a very bumpy surface covered by many large zinc dendrites/ protrusions, and also, some holes and zinc oxide/hydroxide by-products are found (Fig. S10), which can be ascribed to anode corrosion and hydrogen evolution [25, 34]. In sharp contrast, the $\mathrm{Cu}$ nanowire networks-protected $\mathrm{Zn}$ foil electrodes keep a relatively flat surface without the appearance of large zinc dendrites/protrusions. This confirms again that the $\mathrm{Cu}$ nanowire networks effectively protect zinc anodes during zinc plating/stripping processes and improve zinc deposition/stripping efficiency (Fig. S11).

\subsection{Mechanism Investigation}

The underlying mechanisms of protective effects of the $\mathrm{Cu}$ nanowire networks on zinc anodes are investigated in depth. As has been mentioned above, free water and dissolved oxygen-induced corrosion is an important factor causing zinc anode failure. According to linear polarization curves of bare $\mathrm{Zn}$ anodes and $\mathrm{Cu}$ nanowire networks-protected $\mathrm{Zn}$ anodes in $\mathrm{ZnSO}_{4}$ aqueous electrolytes (Fig. 3a), the protected $\mathrm{Zn}$ anodes show higher corrosion potential and 

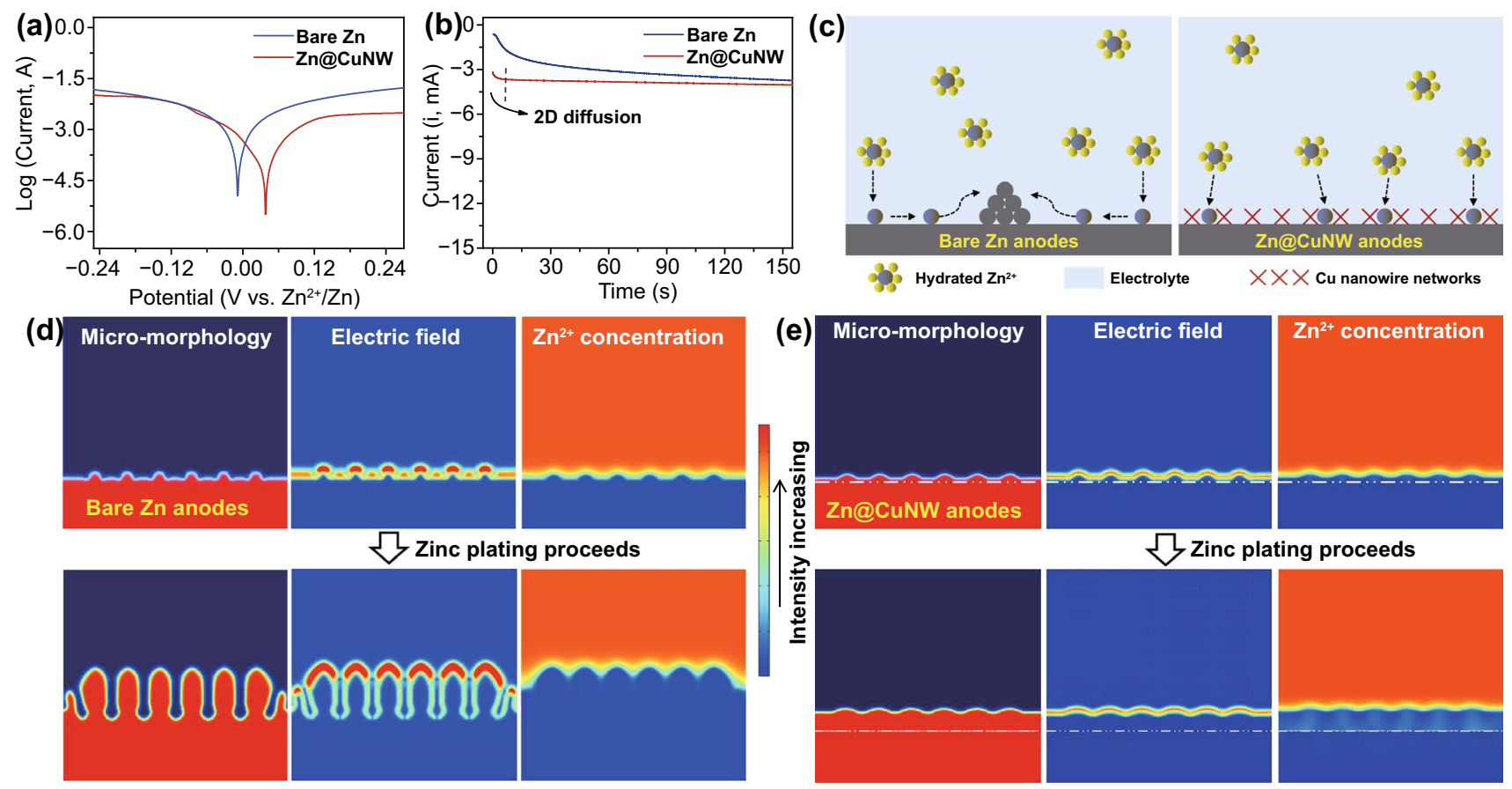

Fig. 3 a Linear polarization curves and $\mathbf{b}$ chronoamperometry curves of the bare Zn anodes and Zn@CuNW anodes. $\mathbf{c}$ Schematic illustration of rampant 2D diffusion behaviors on the bare $\mathrm{Zn}$ anodes (left) and restrained 2D diffusion on the $\mathrm{Zn} @ \mathrm{CuNW}$ anodes. Simulation of surface micro-morphology, electric field and $\mathrm{Zn}^{2+}$ concentration field during the zinc plating process on $\mathbf{d}$ bare $\mathrm{Zn}$ anodes and e Zn@CuNW anodes (the white dash dot lines point out the original position of $\mathrm{Cu}$ nanowire networks/zinc anode interface)

smaller corrosion current, indicating enhanced corrosionresistant ability $[22,46]$. This benefits from the hydrophobic feature of the $\mathrm{Cu}$ nanowire networks, which suppresses the direct contact between free water (with dissolved oxygen) and zinc anodes. Chronoamperometry tests at a constant potential of $-150 \mathrm{mV}$ were performed to investigate $\mathrm{Zn}^{2+}$ diffusion behaviors on anode surface. As shown in Fig. 3b, the current density of the bare $\mathrm{Zn}$ anodes continuously increases over $150 \mathrm{~s}$, revealing a rampant $2 \mathrm{D}$ diffusion of $\mathrm{Zn}^{2+}$ after their adsorption on anode surface [22]. Due to such $2 \mathrm{D}$ diffusion behaviors, $\mathrm{Zn}^{2+}$ ions move to small protrusions (with high specific surface energy), accelerating the formation of large-sized dendrites, as illustrated in Fig. 3c. Differently, the $\mathrm{Cu}$ nanowire networks-protected $\mathrm{Zn}$ anodes display rapidly stabilized current density after the initial $10 \mathrm{~s}$ in their chronoamperometry curve (Fig. 3b). This reflects that lateral movement of $\mathrm{Zn}^{2+}$ on anode surface is constrained by the $\mathrm{Cu}$ nanowire networks. Since porous artificial interface layers on zinc anode surfaces have been widely proved to have the function of guiding $\mathrm{Zn}^{2+}$ flux and deposition sites [31,32,34], it is reasonable to believe that the $\mathrm{Cu}$ nanowire networks with porous nature homogenize
$\mathrm{Zn}^{2+}$ ion concentration distribution and restrict rampant 2D diffusion of $\mathrm{Zn}^{2+}$ on anode surface (Fig. 3c). In fact, as will be discussed below, zincophilic $\mathrm{Cu}$ nanowires promote zinc nucleation/deposition and thus can reduce the number of $\mathrm{Zn}^{2+}$ ions accumulated at anode surface, also helping to restrict 2D diffusion of $\mathrm{Zn}^{2+}$. Besides, unstable electric field on the zinc anode surface during zinc plating/stripping processes is considered as another important factor in causing uneven $\mathrm{Zn}^{2+}$ ion concentration distribution and zinc dendrite growth [21], while for the $\mathrm{Cu}$ nanowire networks-protected $\mathrm{Zn}$ anodes, highly conductive $\mathrm{Cu}$ nanowires may favor the stabilization of surface electric field, thereby inhibiting zinc dendrite $[34,47]$.

The above analysis is verified by COMSOL Multiphysics finite element simulation (Fig. 3d-e). Since the surface of commercial $\mathrm{Zn}$ foil anodes cannot be ideally smooth, and meanwhile, zinc nucleus/clusters inevitably form during the zinc plating process, there are always some tiny protrusions on the zinc anode surface. Due to the "tip effect," a strong localized electric field generates on the tip of these protrusions and then adsorbs $\mathrm{Zn}^{2+}$ through electrostatic forces to deposit on such sites. As the zinc plating process proceeds, 
these tiny protrusions grow into large dendrites (Fig. 3d). However, in the case of introducing the $\mathrm{Cu}$ nanowire networks on the zinc anode surface, nanoscaled $\mathrm{Cu}$ nanowires with large specific surface area are able to reduce local current density and homogenize $\mathrm{Zn}^{2+}$ concentration field (Fig. 3e). As a result, zinc dendrite growth is dramatically inhibited.

Furthermore, the zincophilic feature of $\mathrm{Cu}$ nanowires also plays an important role in optimizing zinc plating/stripping behaviors. As shown in Fig. 4a, electrodeposition of zinc on bare $\mathrm{Zn}$ anodes corresponds to a large nucleation overpotential, but the nucleation overpotential of zinc deposition on $\mathrm{Zn} @ \mathrm{CuNW}$ anodes is almost negligible. Meanwhile, after zinc deposition on Zn@CuNW anodes, metallic Zn and $\mathrm{CuZn}_{5}$ alloy (JCPDS No. 35-1151) are detected from the
$\mathrm{Cu}$ nanowire networks (Figs. $4 \mathrm{~b}$ and S12-S13). These reveal the tendency of zinc deposition on $\mathrm{Cu}$ nanowires [42].

In order to deeply understand zinc deposition behaviors on $\mathrm{Cu}$ nanowires, DFT calculations were conducted. As a baseline, we firstly calculated the binding energy between zinc atom and the (0001) surface of $\mathrm{Zn}$ plates, which is $-0.71 \mathrm{eV}$ (Fig. 4c-d). To probe the interaction between zinc and $\mathrm{Cu}$ nanowires, we modeled $\mathrm{Cu}$ nanowires as a single crystal terminated by four (111) facets, two (110) facets and two (100) facets (Fig. 4e-f) [48, 49]. The most stable zinc adsorption sites on typical facets and edges of the $\mathrm{Cu}$ nanowires are summarized in Fig. 4g-k. Zinc deposition on the (111) facets of the $\mathrm{Cu}$ nanowires corresponds to the binding energy of $-1.30 \mathrm{eV}$, revealing that $\mathrm{Cu}$ nanowires are more zincophilic than $\mathrm{Zn}$ plates. Besides, the calculations point out that $\mathrm{Cu}-\mathrm{Zn}$ solid solutions $\left(\mathrm{CuZn}_{x}\right)$ tend to form during
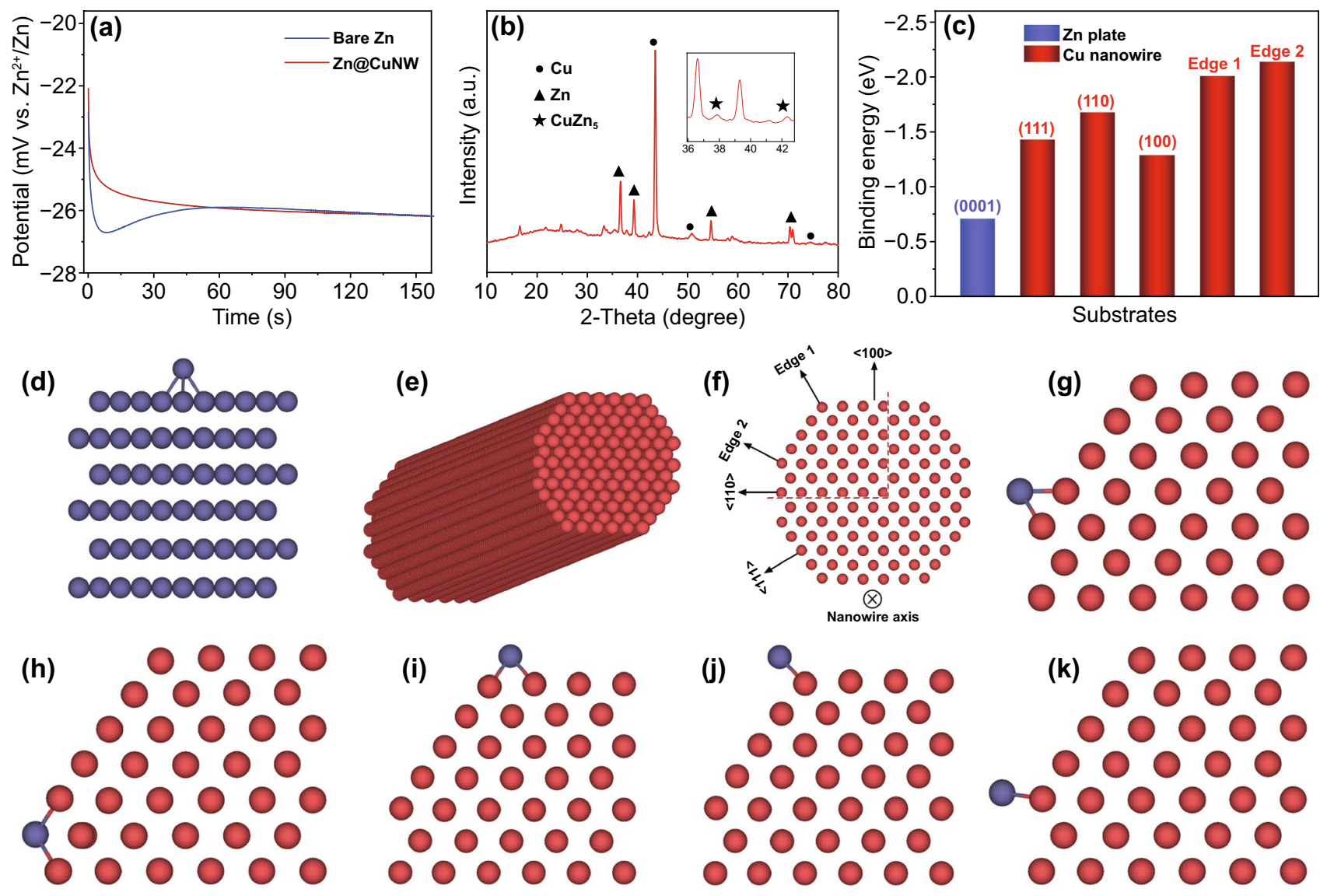

Fig. 4 a Zinc deposition curves at $0.1 \mathrm{~mA} \mathrm{~cm}{ }^{-2}$ on bare $\mathrm{Zn}$ anodes and $\mathrm{Zn@CuNW}$ anodes. b XRD pattern (inset: enlarged zone) of CuNW/

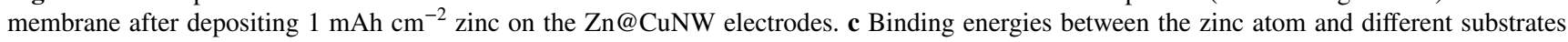
of $\mathrm{Zn}$ plate and $\mathrm{Cu}$ nanowires. $\mathbf{d}$ Calculation model of zinc absorbed on (0001) facet of $\mathrm{Zn}$ plates. $\mathbf{e}$ Bird view and $\mathbf{f}$ atomic model of the singlecrystalline $\mathrm{Cu}$ nanowire. Stable adsorption sites for one zinc atom on various facets and edges of the $\mathrm{Cu}$ nanowires: $\mathbf{g}$ (111) facet, $\mathbf{h}$ (110) facet, (i) (100) facet, $\mathbf{j}$ edge 1 and $\mathbf{k}$ edge 2 . The Lyons blue and brownish red balls are $\mathrm{Zn}$ and $\mathrm{Cu}$ atoms, respectively 

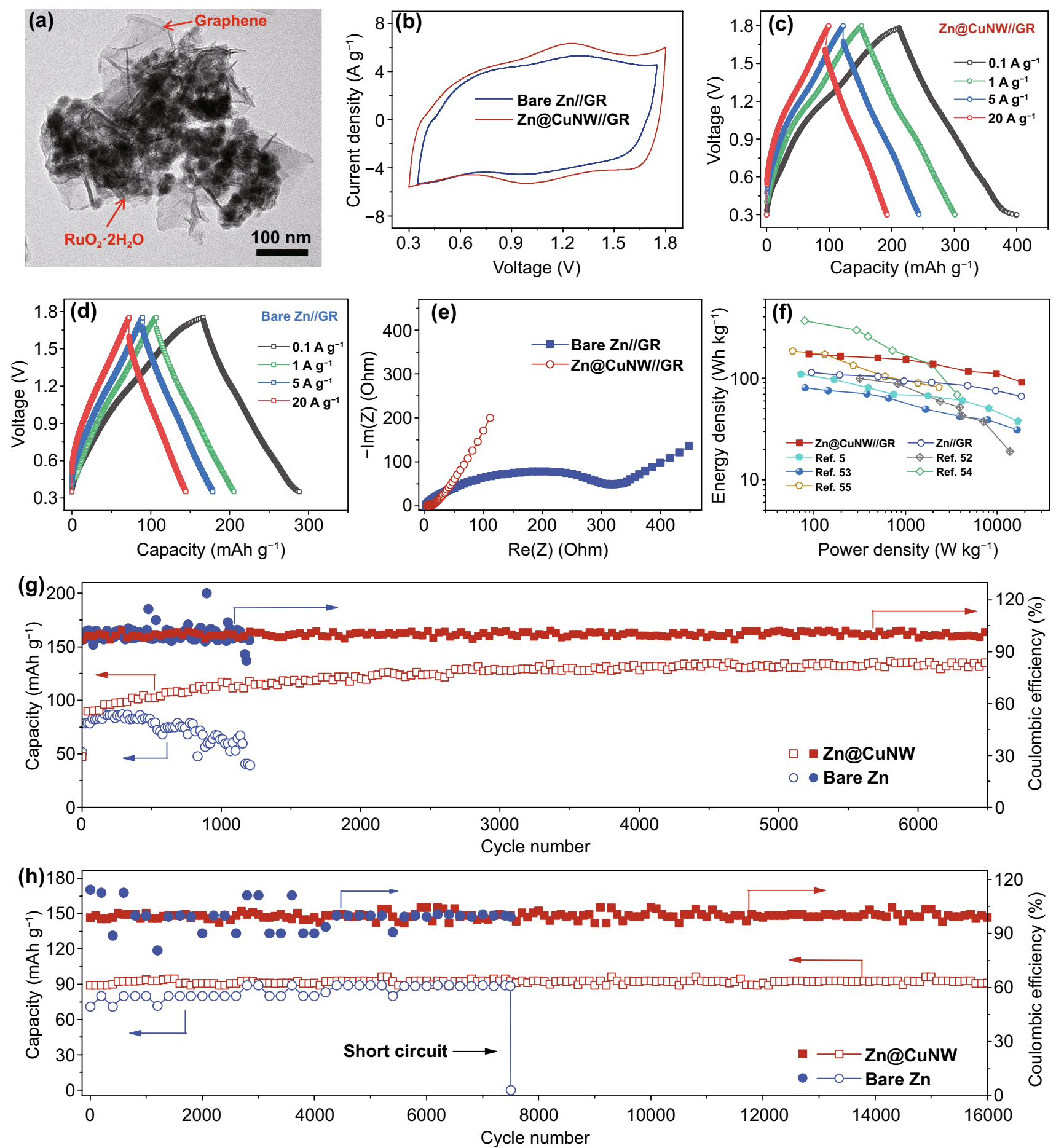

Fig. 5 a TEM image of the GR nanocomposite. Electrochemical properties of the Zn@CuNW//GR and the bare Zn//GR zinc-ion hybrid supercapacitors: b CV curves at $20 \mathrm{mV} \mathrm{s}^{-1}$. c, d GCD curves at different current densities. e EIS spectra. f Ragone plots and long-term cycling behaviors at $\mathbf{g} 5$ and $\mathbf{h} 20 \mathrm{~A} \mathrm{~g}^{-1}$

zinc deposition on high-index facets such as $\mathrm{Cu}(110)$, which strengthens the interaction between zinc and $\mathrm{Cu}$ nanowires to a higher level of $-1.69 \mathrm{eV}$. More importantly, for the $\mathrm{Cu}$ nanowires, their edge sites with un-fully coordinated atoms are found to be highly active for zinc deposition, with binding energy values of $-2.01 \mathrm{eV}$ for the edge $<111,100>$ (i.e., 
the intersection of (111) and (100) facets) and $-2.14 \mathrm{eV}$ for the edge $<111,110>$ (i.e., the intersection of (111) and (110) facets). Therefore, although zinc deposition on the flat facets of $\mathrm{Cu}$ nanowires and on the flat facets of $\mathrm{Cu}$ plates exhibits similar binding energy values (Fig. S14), Cu nanowires with abundant edge sites are more advantageous than $\mathrm{Cu}$ plates as zincophilic materials to induce the uniform nucleation/ deposition of zinc. This well explains that the $\mathrm{Cu}$ nanowire networks-protected zinc anodes exhibit much better electrochemical performance than previously reported $\mathrm{Cu}$ plate-supported zinc anodes (Table S1). Moreover, the easy nucleation/deposition of zinc on $\mathrm{Cu}$ nanowires also contributes to the constrained 2D diffusion of $\mathrm{Zn}^{2+}$ on $\mathrm{Zn} @ \mathrm{CuNW}$ anodes, since it helps to reduce the number of $\mathrm{Zn}^{2+}$ ions accumulated at anode/electrolyte interface.

The long-term stable and high-rate Zn@CuNW anodes enable high-performance Zn-based EES devices. As a demonstration, we assembled zinc-ion hybrid supercapacitors with the $\mathrm{Zn@CuNW}$ anode, GR and $2 \mathrm{M} \mathrm{ZnSO}_{4}$ aqueous electrolyte (Fig. 5). Physicochemical characteristics of the GR nanocomposite are provided in Figs. 5a and S15-S18. In the GR nanocomposite, amorphous $\mathrm{RuO}_{2} \cdot 2 \mathrm{H}_{2} \mathrm{O}$ nanoparticles are loaded on graphene nanosheets. It needs to emphasize that the existence of structural water is essential for ruthenium oxides to store $\mathrm{Zn}^{2+}$ ions through a pseudocapacitive mechanism [50]. For the assembled Zn@CuNW// GR zinc-ion hybrid supercapacitors, they can be charged/ discharged in the voltage window of $0.3-1.8 \mathrm{~V}$ (Figs. $5 \mathrm{~b}$ and S19), corresponding to pseudocapacitive storage of $\mathrm{Zn}^{2+}$ by $\mathrm{RuO}_{2} \cdot 2 \mathrm{H}_{2} \mathrm{O}$ and ion adsorption/desorption on graphene surface [50, 51]. A high discharge capacity of $197 \mathrm{mAh} \mathrm{g}^{-1}$ is achieved (Fig. 5c). In addition, the $\mathrm{Zn} @ \mathrm{CuNW} / / \mathrm{GR}$ zincion hybrid supercapacitors exhibit impressive rate capability, with $99 \mathrm{mAh} \mathrm{g}^{-1}$ discharge capacity at a current density of $20 \mathrm{~A} \mathrm{~g}^{-1}$ (i.e., a charge/discharge cycle only needs $36 \mathrm{~s}$ ). This is because electrochemical energy storage of the Zn@ CuNW//GR zinc-ion hybrid supercapacitors is dominated by fast capacitive behaviors, instead of relatively slow diffusion-controlled behaviors, as discussed in Fig. S20. Electrochemical impedance spectroscopy (EIS) spectra reveal the very small charge transfer resistance during energy storage processes of the $\mathrm{Zn} @ \mathrm{CuNW} / / \mathrm{GR}$ zinc-ion hybrid supercapacitors (Fig. 5e), which well explains the outstanding rate performance. As a result, maximum energy density and power density of the $\mathrm{Zn} @ \mathrm{CuNW} / / \mathrm{GR}$ zinc-ion hybrid supercapacitors are as high as $174 \mathrm{Wh} \mathrm{kg}^{-1}$ and $18 \mathrm{~kW} \mathrm{~kg}^{-1}$, respectively (Fig. 5f), suggesting their better rate capability than zinc-ion batteries and superior charge storage ability than carbon based zinc-ion hybrid supercapacitors [24, 52-54]. Moreover, after 6,500 charge/discharge cycles at 5 $\mathrm{A} \mathrm{g}^{-1}$ and 16,000 cycles at $20 \mathrm{~A} \mathrm{~g}^{-1}$, the Zn@CuNW//GR zinc-ion hybrid supercapacitors do not show obvious decay in capacity, and corresponding coulombic efficiency maintains $\sim 100 \%$ during the long-term cycling tests, indicating superior cyclic stability and high reversibility (Fig. 5g-h). The slight increase in capacity in the initial 1500 cycles in Fig. $5 \mathrm{~g}$ is caused by electrode activation. That is, electrolytes gradually infiltrate into the whole electrode and more electrochemically active materials can participate in electrochemical reactions during repeated charge/discharge processes $[55,56]$.

In sharp contrast, when Zn@CuNW anodes are replaced by bare $\mathrm{Zn}$ anodes, assembled bare $\mathrm{Zn} / / \mathrm{GR}$ zinc-ion hybrid supercapacitors display inferior electrochemical properties, such as narrower working voltage window of $0.35-1.75 \mathrm{~V}$ (note that gas evolution occurs if a wider working voltage window such as $0.3-1.8 \mathrm{~V}$ is applied, which is associated with unstable zinc plating behavior on bare zinc anodes), smaller discharge capacity, notably increased electrochemical impedance and seriously deteriorated cycling performance (Figs. 5b-h and S21-S22). These confirm the superior long-term stability and good rate performance of the Zn@ CuNW anodes in Zn-based EES systems, thereby emphasizing again the validity of using $\mathrm{Cu}$ nanowire networks to protect zinc anodes.

\section{Conclusions}

In summary, zincophilic $\mathrm{Cu}$ nanowire networks were employed to stabilize zinc anodes from multiple aspects. For the $\mathrm{Cu}$ nanowire networks covering on the zinc anode surface, their porous nature guided $\mathrm{Zn}^{2+}$ flux, and meanwhile, their large specific surface area endowed by nanoscale size helps to reduce local current density and homogenize $\mathrm{Zn}^{2+}$ concentration field. Besides, the hydrophobic feature of the $\mathrm{Cu}$ nanowire networks suppressed the direct contact between free water (with dissolved oxygen) and zinc anodes, inhibiting side reactions such as anode corrosion and hydrogen evolution. It was revealed that the facets and edge sites of the $\mathrm{Cu}$ nanowires, especially the latter ones, were highly zincophilic to induce uniform zinc nucleation/deposition. 
The above all aspects work together to alleviate zinc dendrite and side reactions. As a result, the $\mathrm{Cu}$ nanowire networksprotected zinc anodes exhibited an ultralong cycle life of over $2,800 \mathrm{~h}$ and could continuously operate for hundreds of hours even at large charge/discharge currents and areal capacities, remarkably superior to bare zinc anodes and most of currently reported zinc anodes. Moreover, zinc-ion hybrid capacitors were constructed with the $\mathrm{Cu}$ nanowire networksprotected zinc anodes, and high capacity, over 16,000-cycle lifespan and rapid charge/discharge ability were achieved. This work provides new thoughts to realize high-performance zinc anodes for Zn-based EES.

Acknowledgements We would like to thank the financial support from the National Natural Science Foundation of China (No. 52002149), Guangdong Basic and Applied Basic Research Foundation (No. 2020A1515111202) and the fellowship of China Postdoctoral Science Foundation (2020M683186). We also appreciate the constructive comments on theoretical calculations from Huan Duan.

Funding Open access funding provided by Shanghai Jiao Tong University.

Open Access This article is licensed under a Creative Commons Attribution 4.0 International License, which permits use, sharing, adaptation, distribution and reproduction in any medium or format, as long as you give appropriate credit to the original author(s) and the source, provide a link to the Creative Commons licence, and indicate if changes were made. The images or other third party material in this article are included in the article's Creative Commons licence, unless indicated otherwise in a credit line to the material. If material is not included in the article's Creative Commons licence and your intended use is not permitted by statutory regulation or exceeds the permitted use, you will need to obtain permission directly from the copyright holder. To view a copy of this licence, visit http://creativecommons.org/licenses/by/4.0/.

Supplementary Information The online version contains supplementary material available at https://doi.org/10.1007/ s40820-021-00783-4.

\section{References}

1. Y. Jin, L. Zou, L. Liu, M.H. Engelhard, R.L. Patel et al., Joint charge storage for high-rate aqueous zinc-manganese dioxide batteries. Adv. Mater. 31(29), 1900567 (2019). https://doi.org/ 10.1002/adma.201900567

2. G. Fang, J. Zhou, A. Pan, S. Liang, Recent advances in aqueous zinc-ion batteries. ACS Energy Lett. 3(10), 2480 (2018). https://doi.org/10.1021/acsenergylett.8b01426
3. N. Zhang, X. Chen, M. Yu, Z. Niu, F. Cheng et al., Materials chemistry for rechargeable zinc-ion batteries. Chem. Soc. Rev. 49(13), 4203-4219 (2020). https://doi.org/10.1039/C9CS0 0349E

4. L.E. Blanc, D. Kundu, L.F. Nazar, Scientific challenges for the implementation of Zn-ion batteries. Joule 4(4), 771 (2020). https://doi.org/10.1016/j.joule.2020.03.002

5. Y. Li, W. Yang, W. Yang, Z. Wang, J. Rong, G. Wang, C. $\mathrm{Xu}, \mathrm{F}$., Kang, L. Dong, Towards high-energy and anti-selfdischarge Zn-Ion hybrid supercapacitors with new understanding of the electrochemistry. Nano-Micro Letters 13(1) (2021). https://doi.org/10.1007/s40820-021-00625-3

6. F. Wang, O. Borodin, T. Gao, X. Fan, W. Sun et al., Highly reversible zinc metal anode for aqueous batteries. Nat. Mater. 17, 543-549 (2018). https://doi.org/10.1038/ s41563-018-0063-Z

7. M. Zhou, S. Guo, J. Li, X. Luo, Z. Liu et al., Surface-preferred crystal plane for a stable and reversible zinc anode. Adv. Mater. 33(21), 2100187 (2021). https://doi.org/10. 1002/adma.202100187

8. H. Tang, J. Yao, Y. Zhu, Recent developments and future prospects for zinc-ion hybrid capacitors: a review. Adv. Energy Mater. 11(14), 2003994 (2021). https://doi.org/10. 1002/aenm.202003994

9. C. Xu, B. Li, H. Du, F. Kang, Energetic zinc ion chemistry: the rechargeable zinc ion battery. Angew. Chem. Int. Ed. 51(4), 933-935 (2012). https://doi.org/10.1002/anie.201106307

10. D. Chao, C. Zhu, M. Song, P. Liang, X. Zhang et al., A highrate and stable quasi-solid-state zinc-ion battery with novel $2 \mathrm{D}$ layered zinc orthovanadate array. Adv. Mater. 30(32), 1803181 (2018). https://doi.org/10.1002/adma.201803181

11. C. Sun, C. Wu, X. Gu, C. Wang, Q Wang (2021) Interface engineering via Ti3C2Tx MXene electrolyte additive toward dendrite-free zinc deposition. Nano-Micro Lett. 13(1). https:// doi.org/10.1007/s40820-021-00612-8

12. Y. Li, W. Yang, W. Yang, Y. Huang, G. Wang et al., Highperformance zinc-ion batteries enabled by electrochemically induced transformation of vanadium oxide cathodes. J. Energy Chem. 60, 233-240 (2021). https://doi.org/10.1016/j.jechem. 2021.01.025

13. H. Liang, Z. Cao, F. Ming, W. Zhang, D.H. Anjum et al., Aqueous zinc-ion storage in $\mathrm{MoS}_{2}$ by tuning the intercalation energy. Nano Lett. 19(5), 3199-3206 (2019). https://doi.org/ 10.1021/acs.nanolett.9b00697

14. M. Yan, P. He, Y. Chen, S. Wang, Q. Wei et al., Water-lubricated intercalation in $\mathrm{V}_{2} \mathrm{O}_{5} \cdot \mathrm{nH}_{2} \mathrm{O}$ for high-capacity and highrate aqueous rechargeable zinc batteries. Adv. Mater. 30(1), 1703725 (2018). https://doi.org/10.1002/adma.201703725

15. J. Huang, Z. Wang, M. Hou, X. Dong, Y. Liu et al., Polyaniline-intercalated manganese dioxide nanolayers as a highperformance cathode material for an aqueous zinc-ion battery. Nat. Commun. 9, 2906 (2018). https://doi.org/10.1038/ s41467-018-04949-4

16. T. Sun, Z.J. Li, Y.F. Zhi, Y.J. Huang, H.J. Fan et al., Poly(2, 5-dihydroxy-1, 4-benzoquinonyl sulfide) as an efficient cathode for high-performance aqueous zinc-organic batteries. Adv. 
Funct. Mater. 31(16), 2010049 (2021). https://doi.org/10.1002/ adfm.202010049

17. J. Xie, F. Yu, J. Zhao, W. Guo, H.L. Zhang et al., An irreversible electrolyte anion-doping strategy toward a superior aqueous Zn-organic battery. Energy Storage Mater. 33, 283-289 (2020). https://doi.org/10.1016/j.ensm.2020.08.027

18. L. Hu, P. Xiao, L. Xue, H. Li, T. Zhai, The rising zinc anodes for high-energy aqueous batteries. EnergyChem 3(2), 100052 (2021). https://doi.org/10.1016/j.enchem.2021.100052

19. P. Sun, L. Ma, W. Zhou, M. Qiu, Z. Wang et al., Simultaneous regulation on solvation shell and electrode interface for dendrite-free $\mathrm{Zn}$ ion batteries: achieved by a low-cost glucose additive. Angew. Chem. Int. Ed. 60(33), 18247-18255 (2021). https://doi.org/10.1002/anie.202105756

20. W. Lu, C. Zhang, H. Zhang, X. Li, Anode for zinc-based batteries: challenges, strategies, and prospects. ACS Energy Lett. 6(8), 2765-2785 (2021). https://doi.org/10.1021/acsen ergylett.1c00939

21. S.B. Wang, Q. Ran, R.Q. Yao, H. Shi, Z. Wen et al., Lamellananostructured eutectic zinc-aluminum alloys as reversible and dendrite-free anodes for aqueous rechargeable batteries. Nat. Commun. 11, 1634 (2020). https://doi.org/10.1038/ s41467-020-15478-4

22. Z. Zhao, J. Zhao, Z. Hu, J. Li, J. Li et al., Long-life and deeply rechargeable aqueous $\mathrm{Zn}$ anodes enabled by a multifunctional brightener-inspired interphase. Energy Environ. Sci. 12(6), 1938-1949 (2019). https://doi.org/10.1039/c9ee00596j

23. Q. Yang, G. Liang, Y. Guo, Z. Liu, B. Yuan et al., Do zinc dendrites exist in neutral zinc batteries: a developed electrohealing strategy to in situ rescue in-service batteries. Adv. Mater. 31(43), 1903778 (2019). https://doi.org/10.1002/adma.20190 3778

24. L. Dong, X. Ma, Y. Li, L. Zhao, W. Liu et al., Extremely safe, high-rate and ultralong-life zinc-ion hybrid supercapacitors. Energy Storage Mater. 13, 96-102 (2018). https://doi.org/10. 1016/j.ensm.2018.01.003

25. L. Ma, Q. Li, Y. Ying, F. Ma, S. Chen et al., Toward practical high-areal-capacity aqueous zinc-metal batteries: quantifying hydrogen evolution and a solid-ion conductor for stable zinc anodes. Adv. Mater. 33(12), 2007406 (2021). https://doi.org/ 10.1002/adma.202007406

26. Z. Kang, C. Wu, L. Dong, W. Liu, J. Mou et al., 3D porous copper skeleton supported zinc anode toward high capacity and long cycle life zinc ion batteries. ACS Sustain. Chem. Eng. 7(3), 3364-3371 (2019). https://doi.org/10.1021/acssu schemeng.8b05568

27. Y. Zeng, X. Zhang, R. Qin, X. Liu, P. Fang et al., Dendritefree zinc deposition induced by multifunctional CNT frameworks for stable flexible Zn-ion batteries. Adv. Mater. 31(36), 1903675 (2019). https://doi.org/10.1002/adma.201903675

28. Y. Yang, C. Liu, Z. Lv, H. Yang, Y. Zhang et al., Synergistic manipulation of $\mathrm{Zn}^{2+}$ ion flux and desolvation effect enabled by anodic growth of a $3 \mathrm{D} \mathrm{ZnF}_{2}$ matrix for long-lifespan and dendrite-free Zn metal. Adv. Mater. 33(11), 2007388 (2021). https://doi.org/10.1002/adma.202007388
29. K. Zhao, C. Wang, Y. Yu, M. Yan, Q. Wei et al., Ultrathin surface coating enables stabilized zinc metal anode. Adv. Mater. Interfaces 5(16), 1800848 (2018). https://doi.org/10.1002/ admi. 201800848

30. C. Deng, X. Xie, J. Han, Y. Tang, J. Gao et al., A sieve-functional and uniform-porous kaolin layer toward stable zinc metal anode. Adv. Funct. Mater. 30(21), 2000599 (2020). https://doi.org/10.1002/adfm.202000599

31. X. Pu, B. Jiang, X. Wang, W. Liu, L. Dong et al., High-performance aqueous zinc-ion batteries realized by MOF materials. Nano-Micro Lett. 12, 152 (2020). https://doi.org/10.1007/ s40820-020-00487-1

32. L. Kang, M. Cui, F. Jiang, Y. Gao, H. Luo et al., Nanoporous $\mathrm{CaCO}_{3}$ coatings enabled uniform $\mathrm{Zn}$ stripping/plating for long-life zinc rechargeable aqueous batteries. Adv. Energy Mater. 8(25), 1801090 (2018). https://doi.org/10.1002/aenm. 201801090

33. Q. Yang, Y. Guo, B. Yan, C. Wang, Z. Liu et al., Hydrogensubstituted graphdiyne ion tunnels directing concentration redistribution for commercial-grade dendrite-free zinc anodes. Adv. Mater. 32(25), 2001755 (2020). https://doi.org/10.1002/ adma.202001755

34. L. Dong, W. Yang, W. Yang, H. Tian, Y. Huang et al., Flexible and conductive scaffold-stabilized zinc metal anodes for ultralong-life zinc-ion batteries and zinc-ion hybrid capacitors. Chem. Eng. J. 384, 123355 (2020). https://doi.org/10.1016/j. cej.2019.123355

35. Z. Wang, L. Dong, W. Huang, H. Jia, Q. Zhao, Y. Wang, B. Fei, F. Pan, Simultaneously regulating uniform $\mathrm{Zn} 2+$ flux and electron conduction by MOF/rGO interlayers for high-performance $\mathrm{Zn}$ anodes. Nano-Micro Lett 13(1), (2021). https://doi. org/10.1007/s40820-021-00594-7

36. F. Xie, H. Li, X. Wang, X. Zhi, D. Chao et al., Mechanism for zincophilic sites on zinc-metal anode hosts in aqueous batteries. Adv. Energy Mater. 11(9), 2003419 (2021). https://doi. org/10.1002/aenm.202003419

37. Y. Tian, Y. An, C. Liu, S. Xiong, J. Feng et al., Reversible zinc-based anodes enabled by zincophilic antimony engineered MXene for stable and dendrite-free aqueous zinc batteries. Energy Storage Mater. 41, 343-353 (2021). https://doi. org/10.1016/j.ensm.2021.06.019

38. T. Foroozan, V. Yurkiv, S. Sharifi-Asl, R. Rojaee, F. Mashayek et al., Non-dendritic Zn electrodeposition enabled by zincophilic graphene substrates. ACS Appl. Mater. Interfaces 11(47), 44077-44089 (2019). https://doi.org/10.1021/acsami. $9 b 13174$

39. Q. Zhang, J. Luan, L. Fu, S. Wu, Y. Tang et al., The threedimensional dendrite-free zinc anode on a copper mesh with a zinc-oriented polyacrylamide electrolyte additive. Angew. Chem. Int. Ed. 58(44), 15841-15847 (2019). https://doi.org/ 10.1002/anie. 201907830

40. J. Cao, D. Zhang, C. Gu, X. Wang, S. Wang et al., Manipulating crystallographic orientation of zinc deposition for dendrite-free zinc ion batteries. Adv. Energy Mater. 11(29), 2101299 (2021). https://doi.org/10.1002/aenm.202101299 
41. Z. Wang, J. Huang, Z. Guo, X. Dong, Y. Liu et al., A metalorganic framework host for highly reversible dendrite-free zinc metal anodes. Joule 3(5), 1289-1300 (2019). https://doi.org/ 10.1016/j.joule.2019.02.012

42. H. Liu, J. Li, X. Zhang, X. Liu, Y. Yan et al., Ultrathin and ultralight $\mathrm{Zn}$ micromesh-induced spatial-selection deposition for flexible high-specific-energy Zn-ion batteries. Adv. Funct. Mater. 31(48), 2106550 (2021). https://doi.org/10.1002/adfm. 202106550

43. X. Fan, H. Yang, X. Wang, J. Han, Y. Wu et al., Enabling stable $\mathrm{Zn}$ anode via a facile alloying strategy and 3D foam structure. Adv. Mater. Interfaces 8(7), 2002184 (2021). https:// doi.org/10.1002/admi.202002184

44. Z. Cao, X. Zhu, D. Xu, P. Dong, M.O.L. Chee et al., Eliminating $\mathrm{Zn}$ dendrites by commercial cyanoacrylate adhesive for zinc ion battery. Energy Storage Mater. 36, 132-138 (2021). https://doi.org/10.1016/j.ensm.2020.12.022

45. S. Li, J. Fu, G. Miao, S. Wang, W. Zhao et al., Toward planar and dendrite-free $\mathrm{Zn}$ electrodepositions by regulating $\mathrm{Sn}$ crystal textured surface. Adv. Mater. 33(21), 2008424 (2021). https://doi.org/10.1002/adma.202008424

46. M. Abdallah, Ethoxylated fatty alcohols as corrosion inhibitors for dissolution of zinc in hydrochloric acid. Corros. Sci. 45(12), 2705-2716 (2003). https://doi.org/10.1016/S0010938X(03)00107-0

47. C. Shen, X. Li, N. Li, K. Xie, J.G. Wang et al., Grapheneboosted, high-performance aqueous Zn-ion battery. ACS Appl. Mater. Interfaces 10(30), 25446-25453 (2018). https://doi.org/ 10.1021/acsami.8b07781

48. K. Jiang, D. Zhao, S. Guo, X. Zhang, X. Zhu et al., Efficient oxygen reduction catalysis by subnanometer Pt alloy nanowires. Sci. Adv. 3(2), e1601705 (2017). https://doi.org/10.1126/ sciadv. 1601705

49. M.J. Kim, S. Alvarez, Z. Chen, K.A. Fichthorn, B.J. Wiley, Single-crystal electrochemistry reveals why metal nanowires grow. J. Am. Chem. Soc. 140(44), 14740-14746 (2018). https://doi.org/10.1021/jacs.8b08053

50. L. Dong, W. Yang, W. Yang, C. Wang, Y. Li et al., High-power and ultralong-life aqueous zinc-ion hybrid capacitors based on pseudocapacitive charge storage. Nano-Micro Lett. 11, 94 (2019). https://doi.org/10.1007/s40820-019-0328-3

51. S. Wu, Y. Chen, T. Jiao, J. Zhou, J. Cheng et al., An aqueous Zn-ion hybrid supercapacitor with high energy density and ultrastability up to 80000 cycles. Adv. Energy Mater. 9(47), 1902915 (2019). https://doi.org/10.1002/aenm.201902915

52. Y. Lu, Z. Li, Z. Bai, H. Mi, C. Ji et al., High energy-power Znion hybrid supercapacitors enabled by layered B/N co-doped carbon cathode. Nano Energy 66, 104132 (2019). https://doi. org/10.1016/j.nanoen.2019.104132

53. T. Xiong, Z.G. Yu, H. Wu, Y. Du, Q. Xie et al., Defect engineering of oxygen-deficient manganese oxide to achieve highperforming aqueous zinc ion battery. Adv. Energy Mater. 9(14), 1803815 (2019). https://doi.org/10.1002/aenm.20180 3815

54. Q. Chen, J. Jin, Z. Kou, C. Liao, Z. Liu et al., $\mathrm{Zn}^{2+}$ pre-intercalation stabilizes the tunnel structure of $\mathrm{MnO}_{2}$ nanowires and enables zinc-ion hybrid supercapacitor of battery-level energy density. Small 16(14), 2000091 (2020). https://doi.org/ 10.1002/smll.202000091

55. L. Dong, C. Xu, Y. Li, C. Wu, B. Jiang et al., Simultaneous production of high-performance flexible textile electrodes and fiber electrodes for wearable energy storage. Adv. Mater. 28(8), 1675-1681 (2016). https://doi.org/10.1002/adma.20150 4747

56. W. Yang, X. Du, J. Zhao, Z. Chen, J. Li et al., Hydrated eutectic electrolytes with ligand-oriented solvation shells for longcycling zinc-organic batteries. Joule 4(7), 1557-1574 (2020). https://doi.org/10.1016/j.joule.2020.05.018 